\title{
Characterization of Nanoparticles by Mass Spectrometry
}

\section{Qin $\mathrm{Hu}^{*}$}

Department of Chemistry, State University of New York at Buffalo, Natural Science Complex, Buffalo, NY 14260, USA

*Corresponding author: Qin Hu, Department of Chemistry, State University of New York at Buffalo, Natural Science Complex, Buffalo, NY 14260, USA, Tel: 716-416-3032; E-mail: qinhu@buffalo.edu

Received Date: March 15, 2017; Accepted Date: March 16, 2017; Published Date: March 30, 2017

Copyright: (c) 2017 Qin H. This is an open-access article distributed under the terms of the Creative Commons Attribution License, which permits unrestricted use, distribution, and reproduction in any medium, provided the original author and source are credited.

\section{Editorial}

While mass spectrometry has been widely used for decades in biomolecules analyses, the story of mass spectrometric characterization of nanoparticles (NPs) is still short. The mass spectrometry has recently received much attention in the nanoparticle world due to its powerful usefulness especially in elucidating the chemical structures of the smaller-sized NPs $(<5 \mathrm{~nm})$ [1-3]. We are witnessing the major improvements of mass spectrometry that are taking places both in the structural analyses of NPs but also in the mass elucidation of NPs. The advances are mainly coming in the form of improvements in instruments such as advances in ion sources.

Among the most basic criterions of a mass spectrometric technique, the two important factors are the resolution and the sensitivity, of which the former one is increasingly important in identifying the accurate chemical structures of NPs, and the later one is of super significance in elucidating the exact mass of NPs. This need for developing mass spectrometric techniques with better resolution and higher sensitivity and searching for pathways to improve was noted in some important research of the leaders in this field.

An important success in this area is the work by Anderson L. Marsh [1], a leading expert in the mass spectrometric characterization of NPs. He gives an insight into how the matrix assisted laser desorption/ ionization time-of-flight (MALDI-TOF) mass spectrometry functions in the characterization of colloidal platinum NPs. One thing that is becoming clear is that the identification of the chemical structures of NPs by MALDI-TOF mass spectrometry can be achieved easily. However, it has to point out that MALDI-TOF mass spectrometry exhibits lower sensitivity with increasing mass, and fails to capture the larger mass ions; therefore, the elucidation of the exact mass of NPs is challenging. This phenomenon also happens to the characterization of non-heavy metal NPs. A typical example is the MALDI-TOF characterization of the chemical structures of carbon nanoparticles by our group [2]. In our further attempts, we found that the sensitivity of the electrospray ionization quadrupole time-of-flight tandem (ESI-QTOF) mass spectrometry $[3,4]$ towards carbon nanoparticles is much better than MALDI-TOF with more mass ions in high mass range can be captured, but it is still limited to reveal its exact mass information.

To this end, the development of mass spectrometry techniques with improved resolution and sensitivity is highly necessary to advance the accurate characterization of nanoparticles. In addition, the slow and patient examination on the certain mechanical factors that limits the resolution and sensitivity of an existing mass spectrometry technique is of equal importance. Making improvements in the resolution and the sensitivity of an order of magnitude may not be as astonishing as developing a new method, but they are of significant importance.

\section{References}

1. Navin JK, Grass ME, Somorjai GA, Marsh AL (2009) Characterization of colloidal platinum nanoparticles by MALDI-TOF mass spectrometry. Anal Chem 81: 6295-6299.

2. Hu Q, Paau MC, Choi MMF, Zhang Y, Gong X, et al. (2014) Better understanding of carbon nanoparticles via high-performance liquid chromatography-fluorescence detection and mass spectrometry. Electrophoresis 35: 2454-2462.

3. Hu Q, Meng X, Choi MMF, Gong X, Chan W (2016) Elucidating the structure of carbon nanoparticles by ultra-performance liquid chromatography coupled with electrospray ionisation quadrupole timeof-flight tandem mass spectrometry. Anal Chim Acta 911: 100-107.

4. Hu Q, Meng X, Chan W (2016) An investigation on the chemical structure of nitrogen and sulfur codoped carbon nanoparticles by ultraperformance liquid chromatography-tandem mass spectrometry. Anal Bioanal Chem 408: 5347-5357. 